\title{
The more subjects the better in research?
}

\author{
Pieter J. Hoekstra
}

(C) Springer-Verlag 2012

In child and adolescent psychiatry, perhaps more so than in other medical specialties, there can sometimes be an uncomfortable gap between research and clinic. While scientific research is typically aimed at findings obtained from groups of children and adolescents, clinicians are committed to provide optimal assessment and treatment for individual patients and families. And it is true that it is not always straightforward to translate findings from research into clinical practice. For example, when a randomized controlled trial has demonstrated efficacy of a certain treatment for children with a particular disorder, this unfortunately does not guarantee that the treatment benefits each individual child with that disorder. Similarly, research findings related to causes of disorders may not always have relevance for the individual child. Some clinicians may even feel that each patient and family is unique and that group-based research findings offer them little for their clinical practice.

Intriguingly, in the research field of genetics a currently ongoing debate of the pros and cons of an approach directed at finding common genetic polymorphisms associated with a disorder in large groups of patients versus an approach directed at individual pedigrees has similarities with the situation described above. Most of the research efforts aimed at finding genes associated with disorders have so far been performed under the "common variant common disease" assumption. The most straightforward avenue to gene discovery under this assumption is

\section{P. J. Hoekstra $(\square)$}

Department of Psychiatry, University Medical Center

Groningen, University of Groningen, P.O. Box 660,

9700 AR Groningen, The Netherlands

e-mail: p.hoekstra@accare.nl association methodologies, aimed at demonstrating that a particular common allele is overrepresented in the population of affected individuals. Here, the primary objective is to identify genetic polymorphisms that account for common genetic risks for a disorder shared by the population. So far, this approach has failed to reveal definitive findings for the common child and adolescent psychiatry disorders. It has become clear that it is too simplistic to imply that a few genetic loci of moderate effect and intermediate frequency would explain major parts of the disease risk in a population. Rather, two contrasting assumptions have now been put forward, to account for the observed heritability of mental disorders, that is, a very large number of very small-effect common variants (the infinitesimal model) versus a large number of large-effect rare variants (the rare allele model). There are valid arguments for either assumption [1]. The first assumption requires much larger sample sizes than have so far been used in gene-wide association studies in the field of child and adolescent psychiatry. Indeed genome-wide association studies, for example in the field of body mass index have been performed in sample sizes approaching $2,500,000$ subjects [3], which is a far step from the sample sizes so far collected in the field of child and adolescent psychiatry. On the other hand, studies designed under the rare allele model have been able to identify disease-related genes with surprisingly small sample sizes; a gene associated with Tourette's disorder has been identified in a twogeneration pedigree, with ten individuals [2].The latter finding illustrates that there is not only value in looking for common characteristics shared by many individuals with a disorder but that also important knowledge can be gained from a more individual approach, much as is the case with regard to clinical research, where in depth study of individual patients can provide much new information. 
The current issue of European Child and Adolescent Psychiatry contains a good mix of studies that profit from large sample sizes as well as those that provide information from a much smaller sample size. A Taiwanese study reports on individual and peer factors associated with ketamine use among adolescents, based on a survey totaling 9,860 adolescents (Lee et al., this issue). Another population-based study based on a national Norwegian cohort provides evidence that extreme prematurity is associated with increased risk of later mental health problems (Elgen et al. this issue). An anonymous school-based survey about sexuality, health, sexual abuse and onlinerelated sexual victimisation or harassment in a nationally representative sample of 3,432 Swedish high school seniors revealed that a substantial number of youth who seek counseling or psychiatric treatment for mental health problems or problems related to victimisation can be expected to be sexual-minority youth as opposed to heterosexual youth (Priebe et al., this issue). The current issue also contains an informative case study of a young woman with Tourette's disorder who developed Oculogyric crisis, a rare extrapyramidal side effect of aripiprazole (Rizzo et al., this issue) as well interesting studies using relatively small sample sizes (Dereu et al., this issue; Hoek et al., this issue).

\section{References}

1. Gibson G (2012) Rare and common variants: twenty arguments. Nat Rev Genet 13(2):135-145. doi:10.1038/nrg3118 Review

2. Ercan-Sencicek AG, Stillman AA, Ghosh AK, Bilguvar K, O'Roak BJ, Mason CE, Abbott T, Gupta A, King RA, Pauls DL, Tischfield JA, Heiman GA, Singer HS, Gilbert DL, Hoekstra PJ, Morgan TM, Loring E, Yasuno K, Fernandez T, Sanders S, Louvi A, Cho JH, Mane S, Colangelo CM, Biederer T, Lifton RP, Gunel M, State MW (2010) L-Histidine decarboxylase and Tourette's syndrome. N Engl J Med 362(20):1901-1908

3. Speliotes EK et al (2010) Association analyses of 249,796 individuals reveal 18 new loci associated with body mass index. Nat Genet 42(11):937-948 